\title{
BMJ Open The correlation between blood pressure and kidney function decline in older people: a registry-based cohort study
}

\author{
Bert Vaes, ${ }^{1,2}$ Emilie Beke, ${ }^{1}$ Carla Truyers, ${ }^{1}$ Steven Elli, ${ }^{1}$ Frank Buntinx, ${ }^{1,3}$ \\ Jan $Y$ Verbakel, ${ }^{1}$ Geert Goderis, ${ }^{1}$ Gijs Van Pottelbergh ${ }^{1,4}$
}

To cite: Vaes B, Beke $E$, Truyers $\mathrm{C}$, et al. The correlation between blood pressure and kidney function decline in older people: a registry-based cohort study. BMJ Open 2015;5:e007571. doi:10.1136/bmjopen-2015007571

- Prepublication history for this paper is available online. To view these files please visit the journal online (http://dx.doi.org/10.1136/ bmjopen-2015-007571).

Received 1 January 2015 Revised 22 May 2015 Accepted 13 June 2015

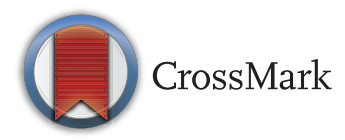

${ }^{1}$ Department of General Practice, Universiteit Leuven (KU Leuven), Leuven, Belgium

${ }^{2}$ Institute of Health and Society, Université catholique de Louvain (UCL), Brussels, Belgium

${ }^{3}$ Department of General

Practice, Maastricht University, Maastricht, The Netherlands

${ }^{4}$ Department of Health and Technology, Leuven University College, Leuven, Belgium

Correspondence to Dr Bert Vaes,

Bert.Vaes@med.kuleuven.be

\section{ABSTRACT}

Objectives: To examine the relation between static and dynamic blood pressure (BP) measurements and the evolution of kidney function in older people, adjusted for the presence of multimorbidity.

Design: Retrospective cohort study during a 10-year time interval (2002-2012) in three age strata of patients aged 60 and older.

Setting: Primary care registration network with 97 general practitioners working in 55 practices regularly submitting collected patient data.

Participants: All patients with at least one BP measurement in 2002 and at least four serum creatine measurements after $2002(\mathrm{n}=8636)$. A modified Charlson Comorbidity Index (mCCl) at baseline was registered. Change in systolic and diastolic BP (DBP) and pulse pressure (PP) from 2002 onwards was calculated. The relation between kidney function evolution and baseline BP and change in BP was examined using linear and logistic regression analysis.

Main outcome measures: The slope of the estimated glomerular filtration rate (eGFR, MDRD, Modification of Diet in Renal Disease equation) was calculated by the ordinal least square method. A rapid annual decline of kidney function was defined as $\geq 3 \mathrm{~mL} / \mathrm{min} / 1.73 \mathrm{~m}^{2} /$ year.

Results: Rapid annual decline of kidney function occurred in 1130 patients (13.1\%). High baseline systolic BP (SBP) and PP predicted kidney function decline in participants aged $60-79$ years. No correlation between baseline BP and kidney function decline was found in participants aged 80 years and older. An annual decline of $\geq 1 \mathrm{~mm} \mathrm{Hg}$ in SBP and PP was a strong risk factor for a rapid annual kidney function decline in all age strata, independent of baseline BP and $\mathrm{mCCl}$. A decline in DBP as also a strong independent predictor in participants aged 60-79 years.

Conclusions: The present study identified a decline in $\mathrm{BP}$ over time as a strong risk factor for kidney function decline in all age strata, adjusted for $\mathrm{mCCl}$ and baseline kidney function and BP.

\section{INTRODUCTION}

Belgium and other western countries are facing a grey epidemic. Furthermore, a

\section{Strengths and limitations of this study}

- The first study that investigated the relation between dynamic blood pressure measurements and kidney function over time in participants aged 60 years and older.

- Large primary care study population representative of the population of Flanders with a long follow-up period.

- Analyses in various age strata were performed in order to detect possibly different patterns due to age.

- The presence of multimorbidity was included in the analyses.

- Lack of mortality data, data on renal replacement therapy, insufficient data on proteinuria/albuminuria and no standardised measurements of creatinine and blood pressure.

- The results are purely descriptive and were not adjusted for time-dependent changes in medication prescription and incident comorbidity.

- Weaknesses inherent to a retrospective design and registry data: possible healthy survivor bias, no information about missing data and loss to follow-up.

'double grey' epidemic is expected, given the proportionally higher increase of persons aged 80 years and older. In 2012, 17.4\% and $5.2 \%$ of the total Belgian population was aged 65 years or older, and 80 years or older, respectively. By 2050, these percentages will rise to $24.5 \%$ and $9.5 \%$, respectively. ${ }^{1}$ This will probably lead to a dramatic increase of chronic diseases and an increased number of patients with multiple comorbidities.

The prevalence of chronic kidney disease (CKD) (estimated glomerular filtration rate (eGFR) $\quad<60 \mathrm{~mL} / \mathrm{min} / 1.73 \mathrm{~m}^{2}$ ) increases with ageing to approximately $10 \%$ at the age of 65 years and to $60 \%$ in persons aged 80 years and older. $^{2-4}$ CKD and especially end-stage renal disease (ESRD) is recognised as an important problem in public health. First, the cost of dialysis per patient per year 
is more than $€ 50000$, and $>1 \%$ of the public health budget of the Belgian government is used to cover these costs. Second, CKD increases the risk of cardiovascular events and mortality. Moreover, many medications cannot be used or need dose adjustment in patients with CKD. ${ }^{5} 6$

Arterial hypertension and cardiovascular disease have been identified both as a cause and as a consequence of $\mathrm{CKD}^{7-9}$ and ESRD. ${ }^{4}$ This has been well studied in the younger population. However, to date, many clinical trials and clinical studies have excluded older persons and especially older persons with multiple chronic conditions. ${ }^{10}$ Furthermore, studies investigating the association between arterial hypertension and the risk of kidney function decline in older persons are scarce. The Cardiovascular Health study ${ }^{11}$ and the Systolic Hypertension in the Elderly Program (SHEP) study $^{8}$ identified baseline BP as a risk factor for kidney function decline in older persons. The Leiden 85 Plus-study ${ }^{12}$ on the other hand, did not find a relation between baseline BP and kidney function decline. It reported a decline in systolic BP (SBP) and diastolic BP (DBP) between ages 85 and 90 years to be related to an accelerated decline of creatinine clearance over time. To date, the relation between the evolution of BP and that of kidney function over time has not been studied in persons aged 60 years and older. Moreover, the impact of concomitant chronic conditions on this relation has not been examined.

Therefore, the aim of this retrospective cohort study within the framework of a large Flemish morbidity registry was to study the relation between static and dynamic $\mathrm{BP}$ measurements and the evolution of kidney function over time in three age strata of participants aged 60 years and older, adjusted for the presence of multimorbidity.

\section{METHODS}

\section{Study design and study population}

Data were obtained from Intego, a Belgian general practice-based morbidity registration network at the Department of General Practice of the University of Leuven. ${ }^{13}$ Intego procedures were approved by the ethical review board of the Medical School of the Catholic University of Leuven ( $\mathrm{N}^{\circ}$ ML 1723) and by the Belgian Privacy Commission (no SCSZG/13/079). Ninety-seven general practitioners (GPs) of 55 practices evenly spread throughout Flanders, Belgium, collaborate in the Intego project. GPs applied for inclusion in the registry. Before acceptance of their data, registration performance was audited using algorithms to compare their results with those of all other applicants. Only the data of the practices with optimal registration performance were included in the database. The Intego GPs prospectively and routinely registered all new diagnoses and new drug prescriptions, as well as laboratory test results and patient information, using computer-generated keywords internally linked to codes.
With specially framed extraction software, new data were encrypted and collected from the GPs' personal computers and entered into a central database. Registered data were continuously updated and historically accumulated for each patient. New diagnoses were classified according to a very detailed thesaurus automatically linked to the International Classification of Primary Care (ICPC-2) and International Statistical Classification of Diseases and Related Health Problems 10th Revision (ICD-10). Drugs were classified according to the WHO's Anatomical Therapeutic Chemical (ATC) classification system.

The present study used Intego data of a 10-year time period from 1 January 2002 to 1 January 2012. First, patients aged 60 years or older in 2002 with a BP measurement registered in 2002 were selected $(n=12$ 904). Second, patients with at least four serum creatine measurements after 2002 were withheld $(\mathrm{n}=8636)$.

\section{Clinical characteristics \\ Blood pressure}

BP measurements registered by the GP in 2002 (baseline or static BP) and yearly thereafter were used. For each year of the study time interval, a single SBP and DBP value was used in the analyses. The average $B P$ of the two lowest values of that year's last three measurements were used. ${ }^{14}$ Pulse pressure (PP) was calculated as the difference between the SBP and the DBP. Categories of baseline $\mathrm{BP}$ measurements were based on previously reported categories. ${ }^{11}$

The slope of the SBP, DBP and PP ( $\mathrm{mm} \mathrm{Hg} /$ year) was calculated for every study participant of whom BP measurements were available for at least 4 years following 2002 ( $\mathrm{n}=7283)$. The slope, or dynamic BP, was calculated according to the ordinal least square method. Patients were divided in categories based on the slope using predefined subgroups of $\leq-3,>-3$ or $\leq-1,>-1$ or $<1$, and $\geq 1 \mathrm{~mm} \mathrm{Hg}$ /year for the SBP and $\leq-1,>-1$ or $<1$ and $\geq 1 \mathrm{~mm} \mathrm{Hg}$ /year for the DBP and the PP.

\section{Kidney function}

Kidney function was expressed as the eGFR calculated with the MDRD (Modification of Diet in Renal Disease) equation. ${ }^{15}$ Baseline eGFR was calculated based on the average serum creatine value of the last two measurements in 2002.

The slope of the eGFR $\left(\mathrm{mL} / \mathrm{min} / 1.73 \mathrm{~m}^{2} /\right.$ year $)$ for every participant was calculated according to the ordinal least square method using all (range 4-50) available eGFR values. A rapid annual decline of kidney function was defined as $\geq 3 \mathrm{~mL} / \mathrm{min} / 1.73 \mathrm{~m}^{2} /$ year; this change is known to be associated with clinically deleterious outcomes. $^{11}$

\section{Comorbidity}

Medical history at baseline of every study participant was registered. The Charlson Comorbidity Index (CCI) includes 19 chronic diseases that are weighted based on 
their association with mortality. ${ }^{16}$ A modified CCI (mCCI) at baseline was calculated for every study participant. ${ }^{17}$ Connective tissue disease could not be reliably assessed from the registry and the differentiation between cancers with or without metastasis, diabetes with or without end organ failure and mild or moderate to severe liver disease could not be made. Consequently all patients with cancers were assigned the same score $(=2)$, as well as all patients with diabetes $(=1)$ and with liver disease $(=1)$.

The prescription of cardiovascular medication at baseline including $\beta$-blockers, ACE inhibitors, angiotensin receptor blockers, calcium antagonists and diuretics was extracted from the database for every study participant.

\section{Data analysis}

Continuous data are presented as the mean and SD. Categorical data are presented as numbers and frequencies. All further analyses were performed in three age strata at baseline: $60-69,70-79$ and $\geq 80$ years.

The correlation between baseline BP measurements and kidney function decline and change in BP and kidney function decline was first explored by calculating ORs with the corresponding 95\% CIs using bivariate and multivariable linear regression analysis and adjusting for age, gender, mCCI at baseline, cardiovascular medication at baseline, time between the first and last eGFR measurement after 2002 ( $\geq$ or $<5$ years) and baseline eGFR (and baseline BP measurements for BP change). Second, the relation between categories of baseline BP measurements or categories of BP change and a rapid annual decline of kidney function was examined with bivariate and multivariable logistic regression analysis.

In order to avoid co-linearity, the correlation coefficients between all covariates were calculated. In case of co-linearity ( $r$-value $>0.90$ ), only one of the two covariates was considered in the multivariate model. Interaction was checked between the slope of the BP measurement and the baseline BP measurement, between the slope of the $\mathrm{BP}$ measurement and the mCCI, and between the baseline BP measurement and the mCCI. If the interaction term was statistically significant $(p<0.05)$, it was kept in the model. A goodnessof-fit test was performed with the ANOVA (analysis of variance) $\mathrm{F}$ test for the linear regression models and the Hosmer-Lemeshow test for the logistic regression models and was reported when the model did not fit the observed data (ANOVA $F$ test $\mathrm{p} \geq 0.05$ and Hosmer-Lemeshow test $\mathrm{p}<0.05)$.

Statistical analyses were performed using SPSS 22.0 (SPSS Inc., Chicago, Illinois, USA) and GraphPad prism 6 (GraphPad Software, San Diego, California, USA).

\section{RESULTS}

In total, 8636 patients from the Intego registry had at least one BP measurement registered in 2002 and at least four serum creatine measurements after 2002. The baseline clinical characteristics of the study population according to their age at baseline are presented in table 1. After baseline, rapid annual decline of kidney function occurred in 1130 patients $(13.1 \%)$ : in $9.4 \%$ $(\mathrm{n}=387)$ of patients aged $60-69$ years, in $15.1 \%(\mathrm{n}=533)$ of patients aged $70-79$ years and in $21.5 \%(n=210)$ of patients aged 80 years or older. Figure 1 presents the prevalence of rapid annual decline of kidney function according to categories of baseline $\mathrm{BP}$ and categories of change in BP. Prevalence of rapid decline of kidney function increased with higher baseline SBP and PP $\left(\chi^{2}\right.$ test, $\mathrm{p}<0.001)$, as well as with increased decline in SBP, DBP and PP $\left(\chi^{2}\right.$ test, $\left.\mathrm{p}<0.001\right)$.

\section{Correlation between SBP and kidney function decline Baseline SBP}

An inverse linear relation was found between baseline SBP and kidney function decline in all age strata, also after adjusting for confounders (table 2). The goodnessof-fit test was not significant in the oldest age stratum (ANOVA F test, $\mathrm{p}=0.23$ ). Categories of higher baseline SBP predicted rapid decline of kidney function in patients aged 60-69 years (adjusted OR 1.9 (95\% CI 1.2 to 3.1) and adjusted OR 2.4 (95\% CI 1.5 to 3.9) for 140 $150 \mathrm{~mm} \mathrm{Hg}$ and $\geq 150 \mathrm{~mm} \mathrm{Hg}$, respectively) (figure 2). In patients aged 70-79 years a trend was seen for the highest category (adjusted OR 1.5 (95\% CI 1.0 to 2.4), $\mathrm{p}=0.052$ ), and no correlation was found in patients aged 80 years or older (Hosmer-Lemeshow test ( $\geq 80$ years), $\mathrm{p}=0.005)$.

\section{Change in SBP}

Figure 3 presents the prevalence of the change in BP for the various age strata. A positive and independent linear relation was found between a change in SBP and a change in kidney function in the oldest two age strata; the more SBP decreased, the more the kidney function decreased in the years after 2002 (table 3). Categories of decreasing SBP showed an increased risk of rapid kidney function decline compared with no change in patients 60-69 years (adjusted OR 1.7 (95\% CI 1.3 to 2.4) and adjusted OR 3.1 (95\% CI 2.0 to 4.6), respectively), in patients 70-79 years (adjusted OR 1.7 (95\% CI 1.3 to 2.3) and adjusted OR 1.9 (95\% CI 1.3 to 2.7), respectively) and in patients aged 80 years and older (adjusted OR 3.3 (95\% CI 1.4 to 8.1 ) and adjusted OR $9.2(95 \%$ CI 1.8 to 46 ), respectively) (figure 4 ). In the oldest age stratum the model was also corrected for the interaction term between change in SBP and baseline mCCI (adjusted OR 1.2 (95\% CI 1.0 to 1.3), $\mathrm{p}=0.026$ ).

\section{Correlation between DBP and kidney function decline Baseline DBP}

No linear relation was found between baseline DBP and kidney function decline (table 2). The goodness-of-fit test for the adjusted model was not significant in the oldest age stratum (ANOVA $\mathrm{F}$ test, $\mathrm{p}=0.64$ ). A trend of 
Table 1 Baseline characteristics of the study population $(n=8636)$

\begin{tabular}{|c|c|c|c|}
\hline & $\begin{array}{l}60-69 \text { Years } \\
n=4128\end{array}$ & $\begin{array}{l}\text { 70-79 Years } \\
n=3530\end{array}$ & $\begin{array}{l}\geq 80 \text { Years } \\
\mathrm{n}=978\end{array}$ \\
\hline Men, n (\%) & $2011(48.7)$ & 1505 (42.6) & $357(36.5)$ \\
\hline Age, mean $\pm S D$ (years) & $64.7 \pm 2.8$ & $74.0 \pm 2.8$ & $83.1 \pm 3.3$ \\
\hline Hypertension, n (\%) & $1855(44.9)$ & $1738(49.2)$ & $451(46.1)$ \\
\hline Systolic blood pressure, mean $\pm \mathrm{SD}(\mathrm{mm} \mathrm{Hg})$ & $134 \pm 14$ & $136 \pm 14$ & $135 \pm 15$ \\
\hline \multicolumn{4}{|l|}{ Systolic blood pressure categories, $\mathrm{n}(\%), \mathrm{mm} \mathrm{Hg}$} \\
\hline$<120$ & $394(9.5)$ & $264(7.5)$ & $70(7.2)$ \\
\hline $120-129$ & $1001(24.2)$ & $779(22.1)$ & $240(24.5)$ \\
\hline 130-139 & $1232(29.8)$ & 1033 (29.3) & $287(29.3)$ \\
\hline $140-149$ & $951(23.0)$ & $872(24.7)$ & $236(24.1)$ \\
\hline$\geq 150$ & 550 (13.3) & $582(16.5)$ & $145(14.8)$ \\
\hline Diastolic blood pressure $(\mathrm{mm} \mathrm{Hg})$, mean $\pm \mathrm{SD}$ & $80 \pm 8$ & $78 \pm 7$ & $76 \pm 7$ \\
\hline \multicolumn{4}{|l|}{ Diastolic blood pressure categories, $\mathrm{n}(\%), \mathrm{mm} \mathrm{Hg}$} \\
\hline$<70$ & $143(3.5)$ & $185(5.2)$ & $90(9.2)$ \\
\hline $70-79$ & $1183(28.7)$ & $1248(35.4)$ & $394(40.3)$ \\
\hline 80-89 & $2240(54.3)$ & $1795(50.8)$ & $445(45.5)$ \\
\hline$\geq 90$ & $562(13.6)$ & $302(8.6)$ & $49(5.0)$ \\
\hline Pulse pressure, mean $\pm \mathrm{SD}(\mathrm{mm} \mathrm{Hg})$ & $54 \pm 11$ & $57 \pm 12$ & $59 \pm 13$ \\
\hline \multicolumn{4}{|l|}{ Pulse pressure, $\mathrm{n}(\%), \mathrm{mm} \mathrm{Hg}$} \\
\hline$<50$ & 1295 (31.4) & $776(22.0)$ & $194(19.8)$ \\
\hline $50-59$ & 1495 (36.2) & $1205(34.1)$ & $308(31.5)$ \\
\hline $60-69$ & $940(22.8)$ & $972(27.5)$ & $282(28.8)$ \\
\hline$\geq 70$ & $398(9.6)$ & 577 (16.3) & $194(19.8)$ \\
\hline Baseline eGFR, mean $\pm S D\left(m L / m i n / 1.73 \mathrm{~m}^{2}\right)$ & $68.8 \pm 13.8$ & $63.2 \pm 14.7$ & $55.4 \pm 15.3$ \\
\hline \multicolumn{4}{|l|}{ Baseline eGFR categories, $\mathrm{n}(\%), \mathrm{mL} / \mathrm{min} / 1.73 \mathrm{~m}^{2}$} \\
\hline$\geq 60$ & $3167(76.7)$ & 2097 (59.4) & $356(36.4)$ \\
\hline $45-59$ & $850(20.6)$ & $1119(31.7)$ & $377(38.5)$ \\
\hline $30-44$ & $97(2.3)$ & $268(7.6)$ & $204(20.9)$ \\
\hline$<30$ & $14(0.3)$ & $46(1.3)$ & $41(4.2)$ \\
\hline Charlson comorbidity index, median (IQR), n (\%) & $3(2-4)$ & $4(3-5)$ & $6(5-7)$ \\
\hline Diabetes & $859(20.8)$ & $800(22.7)$ & $220(22.5)$ \\
\hline Myocardial infarction & $231(5.6)$ & $237(6.7)$ & $80(8.2)$ \\
\hline Heart failure & $135(3.3)$ & $313(8.9)$ & $201(20.6)$ \\
\hline CVA or TIA & $318(7.7)$ & $556(15.8)$ & $229(23.4)$ \\
\hline Peripheral arterial illness & $322(7.8)$ & $404(11.4)$ & $118(12.1)$ \\
\hline Chronic pulmonary disease & $144(3.5)$ & $146(4.1)$ & $36(3.7)$ \\
\hline History of peptic ulcer disease & $381(9.2)$ & $393(11.1)$ & $108(11.0)$ \\
\hline Dementia & $49(1.2)$ & $213(6.0)$ & $87(8.9)$ \\
\hline Liver disease & $172(4.2)$ & $123(3.5)$ & $26(2.7)$ \\
\hline Hemiplegia & $40(1.0)$ & $60(1.7)$ & $20(2.0)$ \\
\hline History of cancer & $523(12.7)$ & $557(15.8)$ & $148(15.1)$ \\
\hline Leukemia & $19(0.5)$ & $24(0.7)$ & $6(0.6)$ \\
\hline Lymphoma & $20(0.5)$ & $27(0.8)$ & $7(0.7)$ \\
\hline Cardiovascular medication, n (\%) & $2061(49.9)$ & $1925(54.5)$ & $554(56.6)$ \\
\hline$\beta$-blockers & $1262(30.6)$ & $1122(31.8)$ & $247(25.3)$ \\
\hline ACE inhibitors & $490(11.9)$ & $509(14.4)$ & $150(15.3)$ \\
\hline Angiotensin receptor blocker & $362(8.8)$ & $300(8.5)$ & $79(8.1)$ \\
\hline Calcium antagonist & $463(11.2)$ & $553(15.7)$ & $167(17.1)$ \\
\hline Diuretic & $905(21.9)$ & 964 (27.3) & $338(34.6)$ \\
\hline
\end{tabular}

predicting rapid decline of kidney function was only seen in the highest category of baseline DBP in patients aged 60-69 years (adjusted OR $2.0 \quad$ (95\% CI 0.99 to 4.0)). The Hosmer-Lemeshow test showed the adjusted model in the oldest patients did not fit the observed data $(\mathrm{p}<0.001)$.

\section{Change in DBP}

An independent and positive linear relation was found between change in DBP and change in kidney function in all age strata (table 3). A decline in DBP predicted rapid decline of kidney function in patients aged 60-69 and 70-79 years (adjusted OR 2.2 (95\% CI 1.7 to 2.9) 

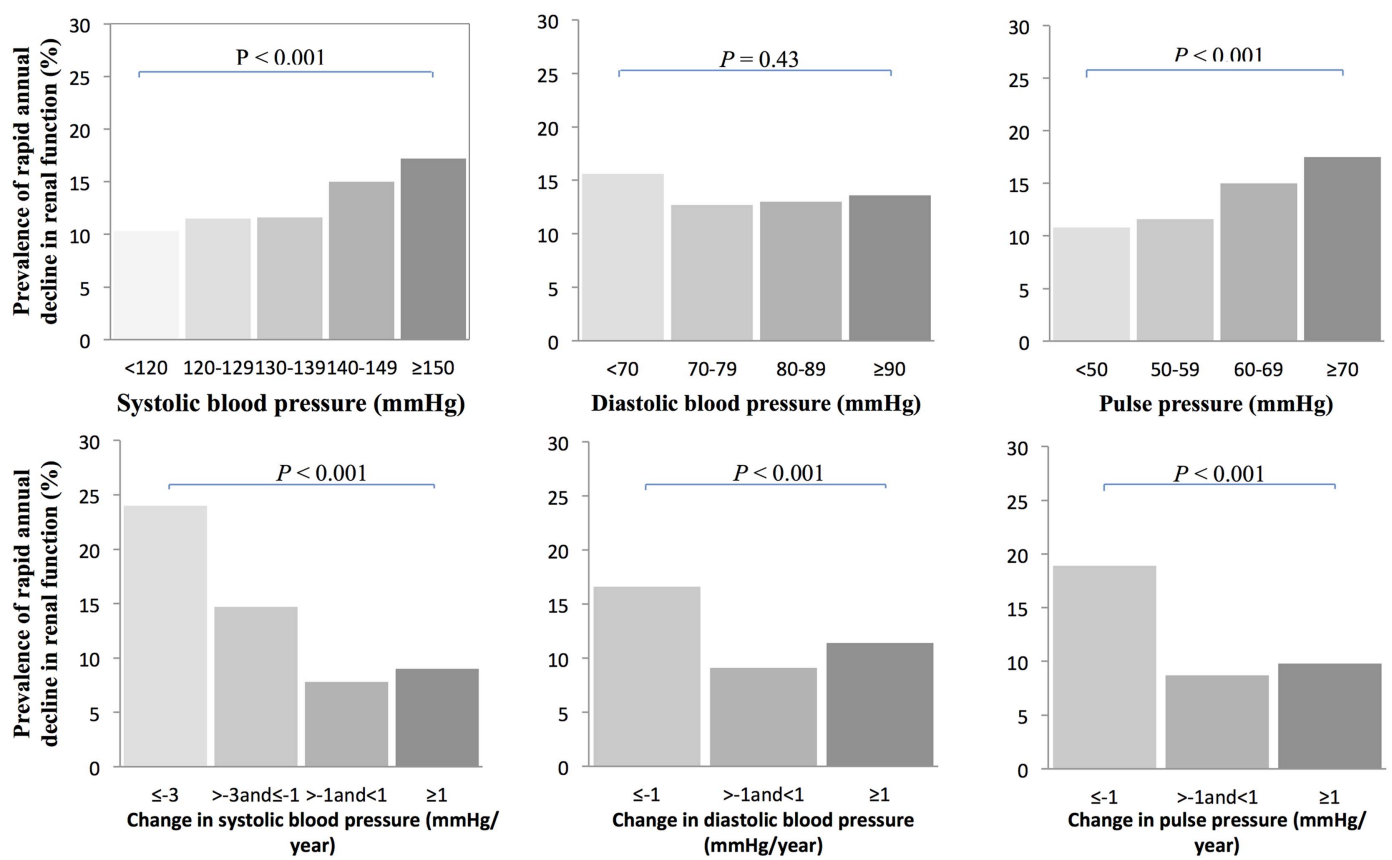

Figure 1 Prevalence of rapid annual decline in kidney function according to different blood pressure measurements.

and adjusted OR 1.4 (1.1 to 1.9), respectively). In the oldest age stratum a trend of higher risk in patients with a decline in DBP was seen (adjusted OR 1.5 (95\% CI 0.99 to 2.4), $\mathrm{p}=0.054$ (Hosmer-Lemeshow test, $\mathrm{p}=0.020)$ ).

\section{Correlation between PP and kidney function decline Baseline PP}

An inverse linear correlation was found between baseline PP and decline in kidney function in all age strata. Only in the oldest age stratum was the goodness-of-fit test for the adjusted model not significant (ANOVA F test, $\mathrm{p}=0.29$ ). The highest categories of baseline PP predicted rapid decline of kidney function in patients aged 60-69 years and aged $70-79$ years (adjusted OR 1.5 (95\% CI 1.0 to 2.2) and adjusted OR 1.4 (95\% CI 1.1 to 2.0).

\section{Change in PP}

A change in PP was independently correlated with change in kidney function in the youngest age group (table 3). The goodness-of-fit test for the adjusted model was not significant in the oldest age stratum (ANOVA F test, $p=0.18)$. Patients in all age strata in the category $\leq-1 \mathrm{~mm} \mathrm{Hg}$ /year change showed a higher risk of rapid annual decline of kidney function compared with patients without change in PP (adjusted OR 2.1 (95\% CI 1.5 to 2.9), adjusted OR 2.8 (95\% CI 1.6 to 4.9 ) and adjusted OR 1.7 (95\% CI 1.1 to 2.8), respectively). In patients $70-79$ years the model was also corrected for the interaction term between change in PP and baseline PP (adjusted OR 1.3 (95\% CI 1.1 to 1.5), $\mathrm{p}=0.009$ ).

\section{DISCUSSION}

In this large retrospective population-based cohort study the relation between static and dynamic BP measurements and kidney function over time in older participants was investigated. The present study confirmed previously found associations between baseline BP measurements and decline of kidney function, but more importantly identified a decline in BP over time as a strong risk factor for kidney function decline in all age strata, independent of the presence of multimorbidity and baseline BP. In people aged 60-69 years, high baseline SBP and PP predicted kidney function decline and a decline in SBP, DBP and PP in the years after baseline were related to a rapid annual decline in kidney function. In patients aged 70-79 years a relation between high baseline SBP and PP and kidney function decline was confirmed, and an association between a decline in SBP, DBP and PP after baseline and a decline in kidney function was seen. In the oldest age stratum, no correlation between baseline BP measurements and kidney function decline was found. A decline in SBP and PP in the years after baseline, however, predicted a rapid annual decline in kidney function in this age group. Moreover, DBP tended to decline.

To date, there is no consensus about which level of BP causes a higher risk of cardiovascular mortality, morbidity or kidney function decline in the elderly population. In the KDOQI/KDIGO guidelines tailored BP control is advised in older persons for the preservation of the kidney function. ${ }^{18}$ This evidence is based on several studies reporting on arterial hypertension as a risk factor for the development of CKD, as well as on the evolution of CKD to ESRD in the global population. The MDRD-trial $^{19}$ studied arterial hypertension as a risk factor for kidney function decline in stage 3 and 4 CKD in a population aged $18-70$ years. They studied the impact of baseline and follow-up BP during 2.2 years on the evolution of kidney function in an intervention (antihypertensive) and in a control group. No effect of high 
Table 2 Baseline BP as a predictor of change in eGFR in $\mathrm{mL} / \mathrm{min} / 1.73 \mathrm{~m}^{2} /$ year (linear regression) ( $\mathrm{n}=8636$ )

\section{0-69 Years \\ $(\mathrm{n}=4128)$ \\ B (95\% Cl)}

Systolic BP (per $10 \mathrm{~mm} \mathrm{Hg}$ )

Adjusted*

Adjusted $\dagger$

$\mathrm{CCl}$ (per point increase)

$\mathrm{CV}$ medication

Baseline eGFR (per mL/min/1.73 $\mathrm{m}^{2}$ )

Diastolic BP (per $10 \mathrm{~mm} \mathrm{Hg}$ )

Unadjusted

Adjusted*

Adjusted $\dagger$

$\mathrm{CCl}$ (per point increase)

CV medication

Baseline eGFR (per $\mathrm{mL} / \mathrm{min} / 1.73 \mathrm{~m}^{2}$ )

Pulse pressure (per $10 \mathrm{~mm} \mathrm{Hg}$ )

Unadjusted

Adjusted*

Adjusted $†$

$\mathrm{CCl}$ (per point increase)

$\mathrm{CV}$ medication

Baseline eGFR (per mL/min/1.73 $\mathrm{m}^{2}$ )

Adusted for age and gender.

Adjusted for gender, age, baseline CCI, baseline CV medication, time between the first and last eGFR measurement after 2002 ( $\geq 5$ years), baseline eGFR

$\mathrm{BP}$, blood pressure; $\mathrm{CCl}$, Charlson Comorbidity Index; CV, cardiovascular; eGFR, estimated glomerular filtration rate.

p Value

$(\mathrm{n}=3530)$

B (95\% Cl)

$-0.14(-0.21$ to -0.066$)$

$-0.14(-0.21$ to -0.064$)$

$-0.11(-0.18$ to -0.034$)$

$-0.15(-0.23$ to -0.061$)$

$-0.28(-0.49$ to -0.073$)$

$-0.014(-0.021$ to -0.006$)$

-0.077 (-0.21 to 0.059$)$

$-0.090(-0.23$ to 0.045$)$

$-0.069(-0.21$ to 0.068$)$

$-0.33(-0.53$ to -0.12$)$

$-0.014(-0.022$ to -0.007$)$

$-0.18(-0.27$ to -0.090$)$

$-0.17(-0.26$ to -0.081$)$

$-0.14(-0.23$ to -0.043$)$

$-0.14(-0.22$ to -0.055$)$

$-0.30(-0.51$ to -0.087$)$

$-0.014(-0.022$ to -0.006$)$
$<0.001$

$<0.001$

0.004

0.001

0.008

$<0.001$

\subsection{7}

0.19

0.33

$<0.001$

0.002

$<0.001$

$<0.001$

$<0.001$

0.004

0.005

$<0.001$
$-0.15(-0.24$ to -0.066$)$
$-0.18(-0.27$ to -0.083$)$

$-0.19(-0.29$ to -0.094$)$

$-0.14(-0.24$ to -0.048$)$

$-0.27(-0.37$ to -0.18$)$

$-0.57(-0.85$ to -0.29$)$

$-0.027(-0.036$ to -0.017$)$

$-0.12(-0.31$ to 0.069$)$

$-0.16(-0.35$ to 0.032$)$

$-0.14(-0.33$ to 0.056$)$

$-0.28(-0.38$ to -0.19$)$

$-0.61(-0.88$ to -0.33$)$

$-0.027(-0.037$ to -0.017$)$

$-0.20(-0.31$ to -0.089$)$

$-0.20(-0.32$ to -0.092$)$

$-0.15(-0.26$ to -0.037$)$

$-0.27(-0.36$ to -0.18$)$

$-0.59(-0.86$ to -0.31$)$

$-0.027(-0.037$ to -0.017$)$

\section{$\geq 80$ Years}

( $\mathrm{n}=978$ )

p Value

B (95\% Cl)

p Value

$<0.001$

$<0.001$

0.003

$<0.001$

$<0.001$

$<0.001$

$-0.26(-0.47$ to -0.043$)$

$-0.27(-0.49$ to -0.055$)$

0.029

$3(-0.20$ to 0.21$)$

$-0.49(-1.1$ to 0.17$)$

$-0.004(-0.028$ to 0.019$)$

0.14

0.73

0.21

0.10

$-0.20(-0.64$ to 0.24$)$

$-0.22(-0.66$ to 0.23$)$

$-0.19(-0.63$ to 0.26$)$

0.001 ( -0.20 to 0.20$)$

0.38

0.17

$<0.001$

$<0.001$

-0.58 ( -1.2 to 0.065$)$

0.41

0.99

$<0.001$

-0.004 ( -0.028 to 0.020$)$

$<0.001$

$-0.26(-0.50$ to -0.025$) \quad 0.031$

$<0.001$

$-0.27(-0.51$ to -0.033$)$

$-0.25(-0.49$ to -0.002$)$

0.006 ( -0.20 to 0.21$)$

$<0.001$

$-0.51(-1.16$ to 0.14$)$

$-0.004(-0.028$ to 0.019$)$

0.026

0.048

0.95

$<0.001$

$<0.001$ 


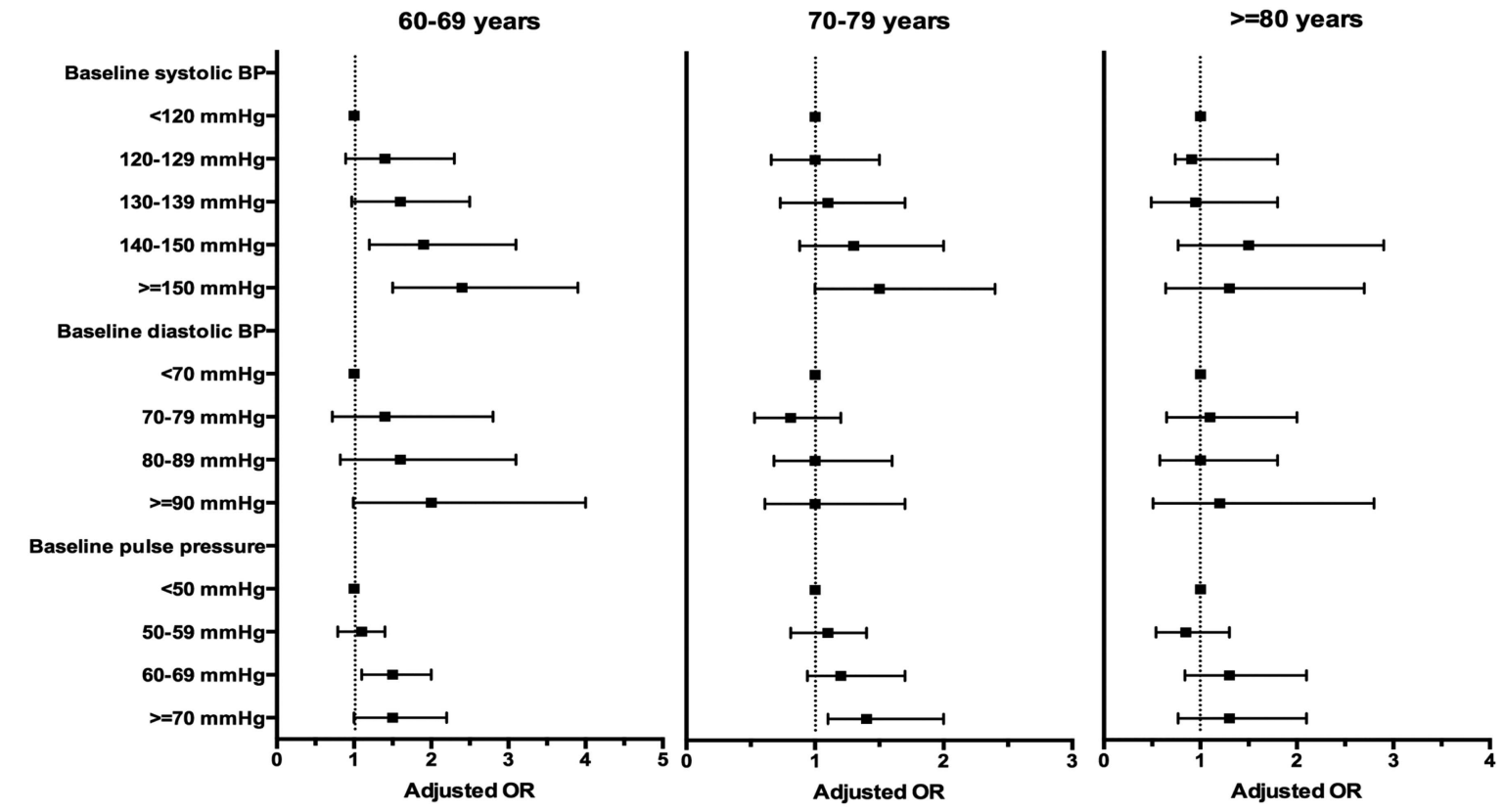

Figure 2 Baseline blood pressure (BP) as a predictor of rapid annual decline in kidney function $\left(\geq 3 \mathrm{~mL} / \mathrm{min} / 1.73 \mathrm{~m}^{2} /\right.$ year) (logistic regression) ( $n=8636$ ). Adjusted for gender, age, baseline Charlson Comorbidity Index, baseline cardiovascular medication, time between the first and last estimated glomerular filtration rate (eGFR) measurement after 2002, baseline eGFR.

BP on kidney function decline was found except for severe proteinuria $>3 \mathrm{~g}$ /day. In a cohort of 332544 men aged $35-57$ years, Klag et $a l^{20}$ found a $22 \%$ higher risk of ESRD in patients with arterial hypertension stage 4, defined as a $\mathrm{SBP}>210 \mathrm{~mm} \mathrm{Hg}$ or a DBP $>120 \mathrm{~mm} \mathrm{Hg}$, compared to that of patients with normal BP. In a large Japanese cohort, Tozawa $e t a l^{21}$ included 98759 patients aged 20-98 years. They found higher baseline SBP and
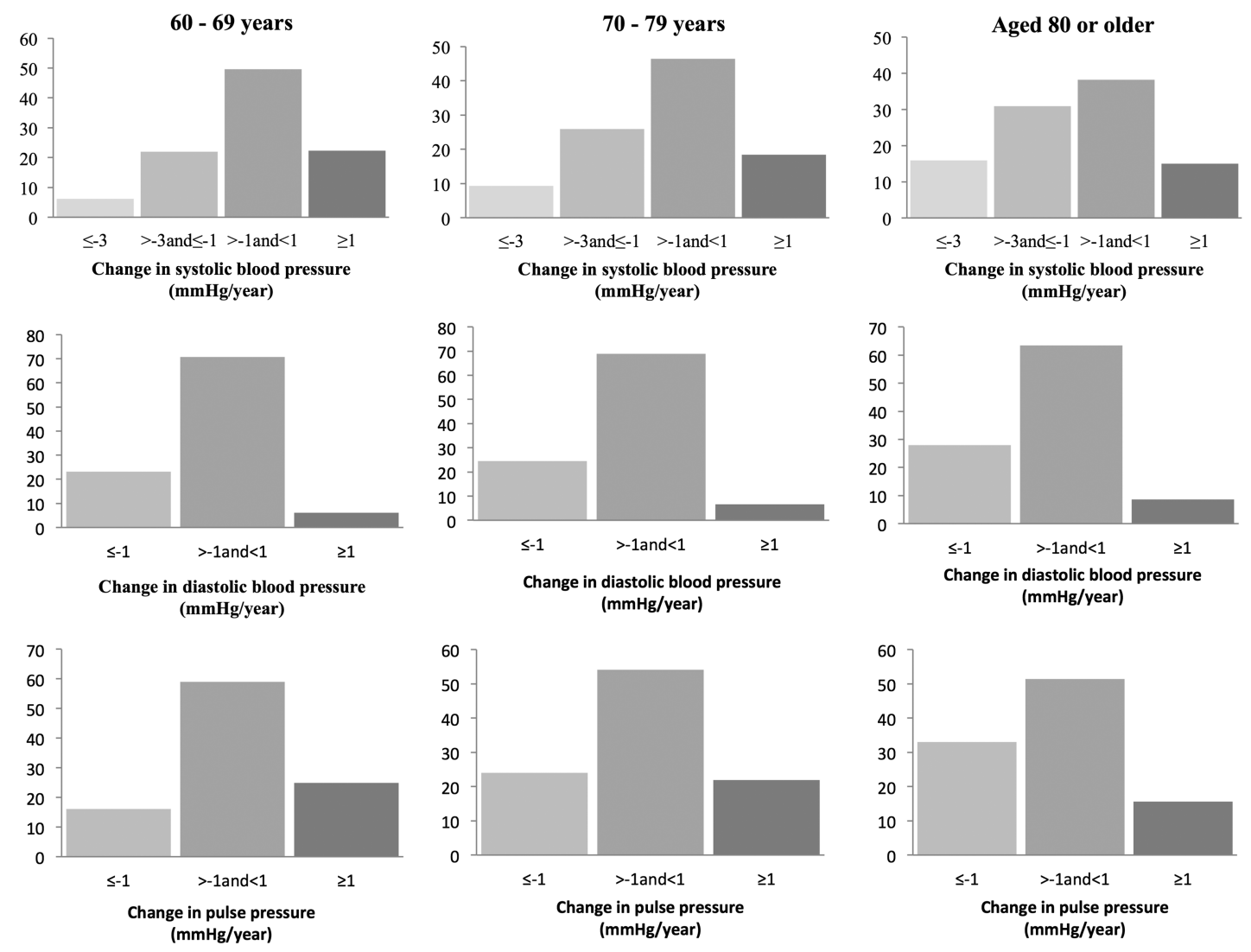

Figure 3 Prevalence (\%) of change in systolic and diastolic blood pressures and pulse pressure in different age strata. 
Table 3 Correlation between change in BP and change in eGFR in $\mathrm{mL} / \mathrm{min} / 1.73 \mathrm{~m}^{2} /$ year (linear regression) $(\mathrm{n}=7283)$

\begin{tabular}{|c|c|c|c|c|c|c|}
\hline & \multicolumn{2}{|l|}{$\begin{array}{l}60-69 \text { Years } \\
(n=3696)\end{array}$} & \multicolumn{2}{|l|}{$\begin{array}{l}\text { 70-79 Years } \\
(n=2933)\end{array}$} & \multicolumn{2}{|l|}{$\begin{array}{l}\geq 80 \text { Years } \\
(n=654)\end{array}$} \\
\hline & $\beta(95 \% \mathrm{Cl})$ & p Value & B (95\% Cl) & p Value & $\beta(95 \% \mathrm{Cl})$ & p Value \\
\hline \multicolumn{7}{|l|}{ Systolic BP change (per mm Hg/year) } \\
\hline Unadjusted & $0.11(0.058$ to 0.16$)$ & $<0.001$ & $0.14(0.080$ to 0.19$)$ & $<0.001$ & $0.17(0.052$ to 0.29$)$ & 0.005 \\
\hline Adjusted $^{*}$ & $0.10(0.052$ to 0.15$)$ & $<0.001$ & $0.13(0.076$ to 0.19$)$ & $<0.001$ & 0.17 (0.048 to 0.29$)$ & 0.006 \\
\hline Adjusted $†$ & $0.030(-0.025$ to 0.086$)$ & 0.28 & $0.069(0.006$ to 0.13$)$ & 0.031 & $1.2(0.34$ to 2.1$)$ & 0.007 \\
\hline Baseline BP pressure (per $10 \mathrm{~mm} \mathrm{Hg}$ ) & $-0.15(-0.22$ to -0.074$)$ & $<0.001$ & $-0.14(-0.23$ to -0.042$)$ & 0.005 & $-0.11(-0.37$ to 0.15$)$ & 0.42 \\
\hline Baseline $\mathrm{CCl}$ (per point increase) & $-0.21(-0.28$ to -0.13$)$ & $<0.001$ & $-0.28(-0.36$ to -0.19$)$ & $<0.001$ & $-0.10(-0.29$ to 0.083$)$ & 0.28 \\
\hline Baseline CV medication & $-0.25(-0.44$ to -0.069$)$ & 0.007 & $-0.50(-0.73$ to -0.26$)$ & $<0.001$ & $-0.61(-1.2$ to -0.023$)$ & 0.042 \\
\hline Baseline eGFR (per mL/min/1.73 $\mathrm{m}^{2}$ ) & $-0.015(-0.021$ to -0.008$)$ & $<0.001$ & $-0.021(-0.030$ to -0.013$)$ & $<0.001$ & $-0.013(-0.035$ to 0.009$)$ & 0.24 \\
\hline $\begin{array}{l}\text { Interaction term 'systolic BP change } \times \text { baseline } \\
\text { systolic BP' }\end{array}$ & NS & NS & NS & NS & $-0.079(-0.14$ to -0.014$)$ & 0.018 \\
\hline \multicolumn{7}{|l|}{ Diastolic BP change (per mm Hg/year) } \\
\hline Unadjusted & $0.16(0.072$ to 0.25$)$ & $<0.001$ & 0.18 (0.073 to 0.29$)$ & 0.001 & $0.38(0.15$ to 0.62$)$ & 0.002 \\
\hline Adjusted* & $0.15(0.064$ to 0.24$)$ & 0.001 & 0.18 (0.069 to 0.28$)$ & 0.001 & 0.37 (0.14 to 0.61$)$ & 0.002 \\
\hline Adjusted $†$ & $0.11(0.008$ to 0.21$)$ & 0.034 & 0.14 (0.019 to 0.25$)$ & 0.023 & $0.42(0.16$ to 0.67$)$ & 0.001 \\
\hline Baseline diastolic BP (per $10 \mathrm{~mm} \mathrm{Hg}$ ) & $-0.082(-0.22$ to 0.054$)$ & 0.24 & $0.57(0.048$ to 1.1$)$ & 0.032 & $0.25(-0.20$ to 0.71$)$ & 0.27 \\
\hline Baseline $\mathrm{CCl}$ (per point increase) & $-0.22(-0.29$ to -0.14$)$ & $<0.001$ & 0.91 (0.029 to 1.8$)$ & 0.043 & $-0.13(-0.32$ to 0.052$)$ & 0.16 \\
\hline Baseline CV medication & $-0.31(-0.50$ to -0.13$)$ & 0.001 & $-0.55(-0.78$ to -0.32$)$ & $<0.001$ & $-0.66(-1.2$ to -0.080$)$ & 0.026 \\
\hline Baseline eGFR (per mL/min/1.73 $\mathrm{m}^{2}$ ) & $-0.015(-0.022$ to -0.008$)$ & $<0.001$ & $-0.021(-0.030$ to -0.013$)$ & $<0.001$ & $-0.015(-0.037$ to 0.007$)$ & 0.18 \\
\hline $\begin{array}{l}\text { Interaction term 'baseline diastolic BP } \times \\
\text { baseline } \mathrm{CCl} \text { ' }\end{array}$ & NS & NS & $-0.15(-0.27$ to -0.041$)$ & 0.008 & NS & NS \\
\hline \multicolumn{7}{|l|}{ Pulse pressure change (per mm Hg/year) } \\
\hline Unadjusted & $0.092(0.031$ to 0.15$)$ & 0.003 & $0.13(0.062$ to 0.20$)$ & $<0.001$ & $0.11(-0.038$ to 0.25$)$ & 0.15 \\
\hline Adjusted* & $0.086(0.025$ to 0.15$)$ & 0.006 & $0.12(0.057$ to 0.19$)$ & $<0.001$ & $0.10(-0.042$ to 0.24$)$ & 0.17 \\
\hline Adjusted $†$ & 0.17 (0.015 to 0.32$)$ & 0.031 & $0.050(-0.025$ to 0.13$)$ & 0.19 & $0.064(-0.10$ to 0.23$)$ & 0.45 \\
\hline Baseline pulse pressure (per $10 \mathrm{~mm} \mathrm{Hg}$ ) & $-0.19(-0.28$ to -0.094$)$ & $<0.001$ & $-0.15(-0.26$ to -0.043$)$ & 0.006 & $-0.052(-0.31$ to 0.20$)$ & 0.69 \\
\hline Baseline $\mathrm{CCl}$ (per point increase) & $-0.20(-0.27$ to -0.13$)$ & $<0.001$ & $-0.28(-0.36$ to -0.19$)$ & $<0.001$ & $-0.13(-0.31$ to 0.059$)$ & 0.18 \\
\hline Baseline CV medication & $-0.27(-0.46$ to -0.091$)$ & 0.003 & $-0.52(-0.75$ to -0.28$)$ & $<0.001$ & $-0.62(-1.2$ to -0.026$)$ & 0.041 \\
\hline Baseline eGFR (per $\mathrm{mL} / \mathrm{min} / 1.73 \mathrm{~m}^{2}$ ) & $-0.015(-0.022$ to -0.008$)$ & $<0.001$ & $-0.021(-0.030$ to -0.013$)$ & $<0.001$ & $-0.014(-0.036$ to 0.008$)$ & 0.22 \\
\hline $\begin{array}{l}\text { Interaction term 'pulse pressure change } \times \\
\text { baseline } \mathrm{CCl} \text { ' }\end{array}$ & $-0.052(-0.096$ to -0.009$)$ & 0.018 & NS & NS & NS & NS \\
\hline
\end{tabular}

${ }^{*}$ Adjusted for age and gender.

†Adjusted for gender, age at baseline, baseline CCl, baseline CV medication, time between the first and last eGFR measurement after 2002, baseline eGFR and baseline BP measurements.

$\mathrm{BP}$, blood pressure; CCI, Charlson Comorbidity Index; CV, cardiovascular; eGFR, estimated glomerular filtration rate; NS, not significant. 


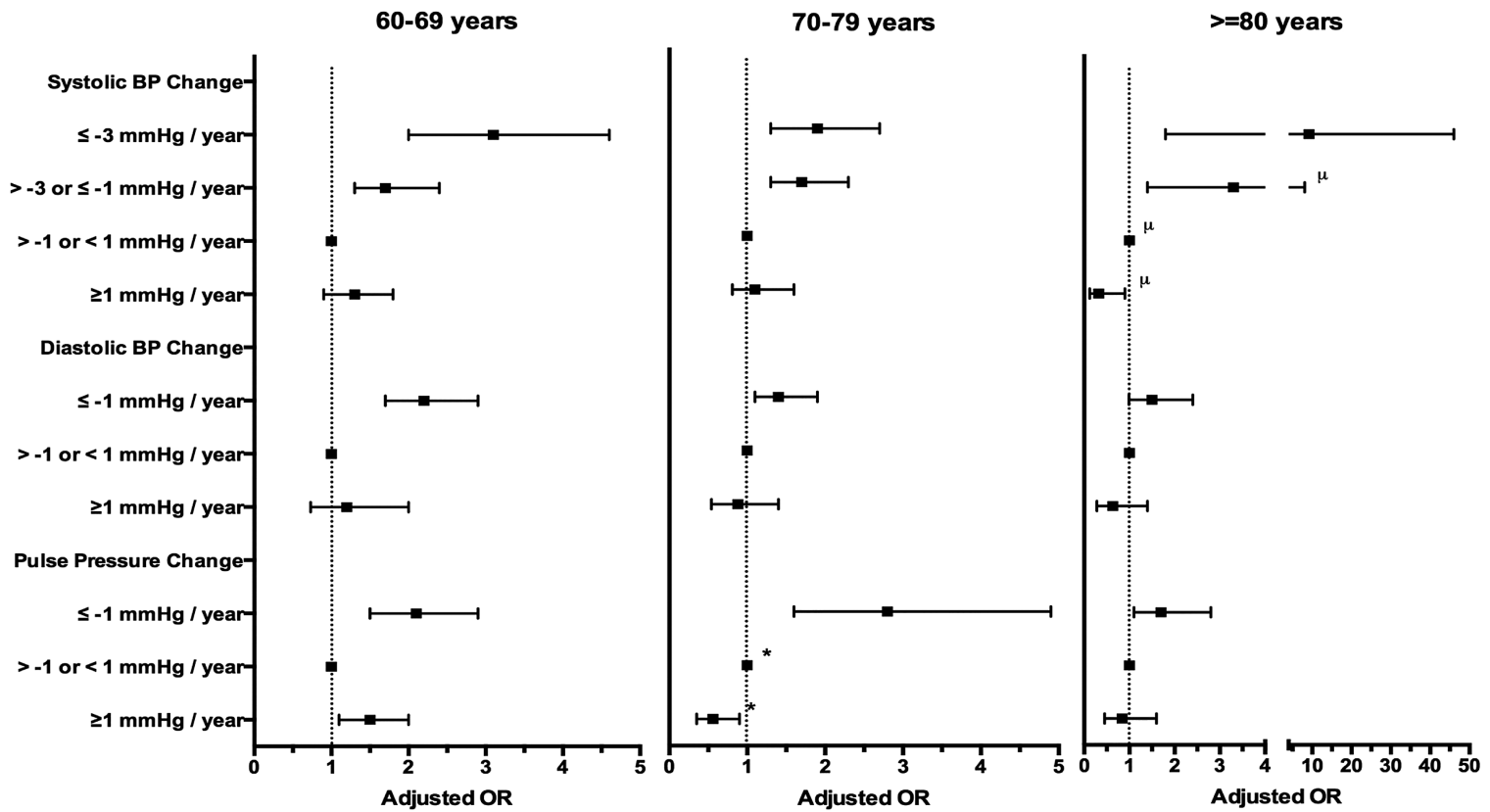

Figure 4 Correlation between change in blood pressure (BP) and a rapid annual decline in kidney function ( $\geq 3 \mathrm{~mL} / \mathrm{min} / 1.73 \mathrm{~m}{ }^{2} /$ year) (logistic regression) ( $n=7283$ ). Adjusted for gender, age at baseline, baseline measurements (systolic or diastolic BP or pulse pressure), baseline Charlson Comorbidity Index $(\mathrm{CCl})$, baseline cardiovascular medication, time between the first and last estimated glomerular filtration rate (eGFR) measurement after 2002, baseline eGFR. ${ }^{\mu}$ Adjusted for interaction term 'systolic BP change $\times$ baseline CCl' (adjusted OR of the interaction term 1.2 (95\% Cl 1.0 to 1.3). *Adjusted for interaction term 'pulse pressure changexbaseline pulse pressure' (adjusted OR of the interaction term 1.3 (95\% $\mathrm{Cl} 1.1$ to 1.5$)$.

DBP showed a significant risk of development of ESRD. Cumulative incidence for ESRD in patients with severe arterial hypertension was $1.7 \%$ vs $0.2 \%$ in patients with a normal BP. Finally, Van Pottelbergh et $a l^{22}$ studied risk factors for ESRD in patients aged 50 years and older in the same Flemish cohort study as the present study did. Baseline arterial hypertension ( $\mathrm{BP} \geq 140 / 90 \mathrm{~mm} \mathrm{Hg}$ ) was found to be a significant risk factor for the development of ESRD (adjusted HR 1.25 (95\% CI 1.22 to 1.28)).

Studies investigating the impact of BP on the evolution of kidney function in older persons are extremely rare. Some studied the risk of baseline BP for kidney function decline. Rifkin et $a l^{11}$ found baseline SBP to have the strongest association with rapid annual kidney function decline in persons aged 65 years or older, with $14 \%$ increased hazard of rapid decline per $10 \mathrm{~mm} \mathrm{Hg}$, independent of other BP measurements. The SHEP-trial ${ }^{8}$ used their placebo-arm to study the relation between baseline BP and a yearly incident increase of serum creatine $(\geq 0.4 \mathrm{mg} / \mathrm{dL})$. They found higher baseline SBP increased the relative risk of kidney function decline. A positive trend was found for DBP, however, without correction for comorbidity. The relation between baseline BP and the evolution of SBP, DBP and PP and kidney function decline in a 5-year time interval has only been studied in patients aged 85 years and older. ${ }^{12}$ The Leiden $85+$ study reported that elevated baseline SBP and DBP did not influence the annual decline in renal function in the oldest individuals. However, DBP $<70 \mathrm{~mm} \mathrm{Hg}$ and a decline in SBP or DBP was related to an accelerated decline of creatinine clearance over time. The present study results are in line with these findings.

The deleterious effect of higher baseline BP measurements on the evolution of the kidney function in persons up to 80 years was confirmed by the present study. Furthermore, intervention trials have shown that BP lowering prevents the need for renal replacement therapy up to the age of 70, independent of renal function at baseline. ${ }^{23}$ The question remains how to explain the observations of the present study that identified a decline of $\geq 1 \mathrm{~mm} \mathrm{Hg}$ /year SBP, DBP or PP as a predictor of kidney function decline, not only in the oldest old, as shown in the Leiden 85+ Study, but also in persons aged 60-79 years old. A decline in BP may lead to chronic hypoperfusion of the kidney, causing the kidney function to deteriorate. The cause of BP decline remains unclear. Underlying heart failure with lowered cardiac output could be a reason. ${ }^{24}$ On the other hand, BP control which is too strict or the effects of antihypertensive medications such as RAAS system inhibitors, which influence intra-renal BP and renal perfusion could be responsible. The analyses were corrected for baseline morbidities, including the presence of heart failure and history of myocardial infarction, cardiovascular medication and baseline BP and kidney function. However, possible changes in medication intake and occurrence of new morbidities in the years after baseline could also be underlying the observed relationship. Furthermore, the results of the present study should be interpreted with caution, since they originate from an 
observational registry-based cohort study. However, they do provide a realistic reflection of every-day practice. Future analyses should further clarify the findings of this study before it could lead to an adaptation or refinement of the current guidelines.

This study is the first that investigated the relation between dynamic BP measurements and kidney function over time in participants aged 60 years and older. The major strengths of this study are its large primary care study population representative of the population of Flanders and the long follow-up period. ${ }^{13}$ Because of the large number of patients included in the study we were able to perform the analyses in various age strata in order to detect possibly different patterns due to age. This study is also the first that included the presence of multimorbidity in the analyses.

However, the study is limited in that we had neither mortality data, nor data on the start of renal replacement therapy. Second, the MDRD equation has several weaknesses as a proxy for the real kidney function. For example, loss of muscle mass in older people may falsely give reduced estimates of renal function. However, this widely used equation to estimate the eGFR corrects for age that can act as a proxy for muscle mass. Since we were interested in the evolution of kidney function in the same population, a change in equation does not affect model outcomes. Third, no data related to proteinuria or albuminuria could be used in the analyses, because they were only available for a limited number of patients. Using these limited albuminuria and proteinuria data would have caused substantial selection bias. Fourth, not all creatinine values were measured by the same laboratory or by the same creatinine assay due to the design of the database, which collects data from practices throughout Flanders. However, all Belgian laboratories are subject to quality control measures, ${ }^{25}$ which limited the analytical differences among the laboratories. Fifth, BP measurements were not standardised, but were reported by the GP as measured with his/her own BP device. Furthermore, the variability in $\mathrm{BP}$ and creatinine measurements in routine clinical practice may have affected the study findings.

\section{CONCLUSION}

This large retrospective, registry-based cohort study investigated the relation between static and dynamic BP measurements and the evolution of kidney function, independent of the presence of multimorbidity. Previously found associations between baseline BP measurements and decline of kidney function in older persons were confirmed, but more importantly a decline in BP over time was identified as a strong risk factor for kidney function decline in all patients aged 60 years and older, independent of the presence of multimorbidity and baseline BP.

Contributors BV, EB and GVP performed the analyses and wrote the manuscript. CT and SE extracted the data. FB is responsible for the study concept, design and acquisition of participants and data. All authors participated in the interpretation of the data.

Funding Intego is funded on a regular basis by the Flemish Government (Ministry of Health and Welfare) and by the Belgian National Institute for Health and Disability Insurance on a contractual basis (ACHIL project). This work would not have been possible without the collaboration of all general practitioners in the Intego network. We hereby state the independence of researchers from funders.

Competing interests All authors have completed the ICMJE uniform disclosure form at http://www.icmje.org/coi_disclosure.pdf and declare: no support from any organisation for the submitted work; no financial relationships with any organisations that might have an interest in the submitted work in the previous 3 years; no other relationships or activities that could appear to have influenced the submitted work.

Ethics approval Intego procedures were approved by the ethical review board of the Medical School of the Catholic University of Leuven ( $\mathrm{N}^{\circ} \mathrm{ML} 1723$ ) and by the Belgian Privacy Commission (no SCSZG/13/079).

Provenance and peer review Not commissioned; externally peer reviewed.

Data sharing statement All authors had full access to all of the data (including statistical reports and tables) in the study and can take responsibility for the integrity of the data and the accuracy of the data analysis.

Open Access This is an Open Access article distributed in accordance with the Creative Commons Attribution Non Commercial (CC BY-NC 4.0) license, which permits others to distribute, remix, adapt, build upon this work noncommercially, and license their derivative works on different terms, provided the original work is properly cited and the use is non-commercial. See: http:// creativecommons.org/licenses/by-nc/4.0/

\section{REFERENCES}

1. http://statbel.fgov.be

2. Van Pottelbergh G, Bartholomeeusen S, Buntinx F, et al. The prevalence of chronic kidney disease in a Flemish primary care morbidity register. Age Ageing 2012;41:231-3.

3. Coresh J, Selvin E, Stevens LA, et al. Prevalence of chronic kidney disease in the United States. JAMA 2007;298:2038-47.

4. U.S. Renal Data System, USRDS 2013 Annual Data Report: Atlas of chronic kidney disease and end-stage renal disease in the United States. Bethesda, MD: National Institutes of Health, National Institute of Diabetes and Digestive and Kidney Diseases, 2013.

5. Go AS, Chertow GM, Fan D, et al. Chronic kidney disease and the risk of death, cardiovascular events, and hospitalization. $N$ Engl J Med 2004;351:1296-305.

6. Shastri S, Katz R, Rifkin DE, et al. Kidney function and mortality in octogenarians: cardiovascular Health Study All Stars. J Am Geriatr Soc 2012;60:1201-7.

7. [No authors listed]. 1995 update of the working group reports on chronic renal failure and renovascular hypertension. National High Blood Pressure Education Program Working Group. Arch Intern Med 1996;156:1938-47.

8. Mailloux LU, Levey AS. Hypertension in chronic renal disease. Am J Kidney Dis 1998;32:S120-41.

9. Walker WG. Hypertension-related renal injury: a major contributor to end-stage renal disease. Am J Kidney Dis 1993;22:164-73.

10. Crome $P$, Lally $F$, Cherubini $A$, et al. Exclusion of older people from clinical trials: professional views from nine European countries participating in the PREDICT study. Drugs Aging 2011;28:667-77.

11. Rifkin DE, Katz R, Chonchol M, et al. Blood pressure components and decline in kidney function in community-living older adults: the Cardiovascular Health Study. Am J Hypertens 2013;26:1034-44.

12. van Bemmel T, Woittiez K, Blauw GJ, et al. Prospective study of the effect of blood pressure on renal function in old age: the Leiden 85-Plus Study. J Am Soc Nephrol 2006;17:2561-6.

13. Truyers $\mathrm{C}$, Goderis $\mathrm{G}$, Dewitte $\mathrm{H}$, et al. The Intego database: background, methods and basic results of a Flemish general practice-based continuous morbidity registration project. BMC Med Inform Decis Mak 2014;14:48.

14. Goderis G, Van Pottelbergh G, Truyers C, et al. Long-term evolution of renal function in patients with type 2 diabetes mellitus: a registrybased retrospective cohort study. BMJ Open 2013;3:e004029.

15. Levey AS, Coresh J, Greene T, et al. Expressing the modification of diet in renal disease study equation for estimating glomerular 
filtration rate with standardized serum creatinine values. Clin Chem 2007:53:766-72.

16. Charlson ME, Pompei $\mathrm{P}$, Ales $\mathrm{KL}$, et al. A new method of classifying prognostic comorbidity in longitudinal studies: development and validation. J Chronic Dis 1987;40:373-83.

17. Boeckxstaens $\mathrm{P}$, Vaes $\mathrm{B}$, Legrand $\mathrm{D}$, et al. The relationship of multimorbidity with disability and frailty in the oldest patients: a cross-sectional analysis of three measures of multimorbidity in the BELFRAIL cohort. Eur J Gen Pract 2015;21:39-44.

18. No authors listed]. Chapter 3: management of progression and complications of CKD. Kidney Int Suppl 2013;3:73-90.

19. Klahr S, Levey AS, Beck GJ, et al. The effects of dietary protein restriction and blood-pressure control on the progression of chronic renal disease. N Engl J Med 1994;330:877-84.

20. Klag MJ, Whelton PK, Randall BL, et al. Blood pressure and end-stage renal disease in men. N Engl J Med 1996;334:13-18.
21. Tozawa M, Iseki $\mathrm{K}$, Iseki C, et al. Blood pressure predicts risk of developing end-stage renal disease in men and women. Hypertension 2003;41:1341-5.

22. Van Pottelbergh G, Bartholomeeusen S, Buntinx F, et al. The evolution of renal function and the incidence of end-stage renal disease in patients aged $\geq 50 y e a r s$. Nephrol Dial Transplant 2012;27:2297-303.

23. Maschio G, Alberti D, Janin G, et al. Effect of the angiotensin-converting-enzyme inhibitor benazepril on the progression of chronic renal insufficiency. The Angiotensin-Converting-Enzyme Inhibition in Progressive Renal Insufficiency Study Group. N Engl J Med 1996;334:939-45.

24. Nichols WW, O'Rourke M, eds. McDonald's blood flow in arteries, theoretical, experimental and clinical principals. Edward Arnold, 1998.

25. https://www.wiv-isp.be/QML/index_nl.htm 